\title{
Modelización de relacio- nes entre magnitudes geométricas en un entorno enriquecido con TICs: actividades para la escuela secundaria, diseñadas en un grupo colaborativo
}

Autores: Valeria Borsani, Mara Cedrón, Rosa Cicala, Enrique Di Rico, Betina Duarte, Carmen Sessa e-mail:valeria.borsani@unipe.edu.ar; mara.cedron@unipe.edu.ar; rosa.cicala@unipe.edu.ar; enrique.dirico@unipe.edu.ar; betina.duarte@ unipe.edu.ar; carmen.sessa@unipe.edu.ar Dirección: Camino Centenario 2565. (B1897AVA) Gonnet, Buenos Aires, Argentina Lugar de trabajo: UNIPE (Universidad Pedagógica) Teléfono: (0221) 484.2697/ 484.4521

\section{Resumen}

Presentamos aquí avances de nuestra investigación que aborda el problema didáctico de integrar las TICs en las clases de matemática de nivel secundario. En este artículo nos referiremos a la instancia del diseño de actividades para el aula en torno a la modelización de relaciones entre magnitudes geométricas. Disponer de una modelización intramatemática en un entorno de geometría dinámica para el estudio de la variación de magnitudes asociadas a la situación, nos permiten plantear, entre otras cuestiones: condiciones que contribuyen en el aula a la distinción entre una situación geométrica y su modelo dinámico; la incidencia de algunas características del funcionamiento del programa en el trabajo matemático de los estudiantes y, finalmente, la configuración del área de trabajo de un entorno de geometría dinámica como variable didáctica.

Palabras clave: estudio variacional, magnitudes geométricas, entornos de geometría dinámica, escuela secundaria, aprendizaje y enseñanza potenciados por TIC. 


\begin{abstract}
We present some of our research progress that address the didactical problem of integrating digital technologies in mathematics classes at the secondary level.

We will refer to the instance of collaborative design for classroom activities around the study of geometrical magnitudes variation in a dynamical geometry environment. Among other issues, we set up: conditions to enable the distinction between a geometrical situation and its dynamical model in mathematical classroom, the emergence of a relationship between the logic of the software performance and the mathematical work that students can develop and, the workspace configuration of a dynamical geometry environment as a didactical variable.
\end{abstract}

Keywords: variational study, geometric magnitudes, dynamical geometry environment, high school, teaching and learning enhanced by TIC. 


\section{Presentación del problema y referencias teóricas que lo enmarcan}

El presente trabajo se sitúa en el marco de una investigación que aborda el problema didáctico de integrar TICs en las clases de matemática de nivel secundario desde la perspectiva de la Didáctica de la Matemática, en un equipo que incorpora docentes de escuela secundaria en ejercicio. ${ }^{(1)}$

En el campo de la investigación en educación matemática se sostiene, desde hace más de quince años, que el trabajo con nuevas herramientas - la calculadora y/o la computadora - trae aparejado un cambio en el tipo de conocimiento que se produce (Balacheff, 2000; Artigue 2002; Lagrange, 2000; Hoyles, Noss y Kent, 2004; Hoyles y Lagrange, 2010).

Más recientemente, Artigue (2013) señala la necesidad de considerar algunos obstáculos que provienen, a su entender, de la incorporación ingenua de la tecnología. Nos importa recuperar algunos de sus señalamientos, tales como:

- La poca sensibilidad a cuestiones instrumentales, la tendencia a ver la tecnología como una herramienta transparente, impidiéndonos pensar las combinaciones originales de conocimientos matemáticos y tecnológicos que sostienen las génesis instrumentales y cuyos aprendizajes se deben organizar.

- La subestimación de la complejidad del trabajo del docente en entornos tecnológicos, y de las competencias nuevas que este trabajo necesita; la subestimación de su trabajo de mediación, impidiéndonos pensar prácticas de formación capaces de sostener eficazmente su desarrollo profesional.

En relación con la incorporación de la tecnología al trabajo matemático de los alumnos Balacheff caracteriza el cambio que se produce:

La introducción del software educativo, del tipo que sea, hace que la situación de enseñanza y aprendizaje sea mucho más compleja desde el punto de vista didáctico porque un sistema informático es, ante todo, la materialización de una tecnología simbólica. Esta particularidad juega un papel clave en dos sentidos:

- Por una parte, modificando el objeto de enseñanza como resultado del proceso de transposición computacional.

- Por otro lado, modificando las relaciones que uno puede tener con dichos objetos o el tipo de problema que resulta relevante o de interés. (Balacheff, 2000:106)

Recuperando estos aportes en el marco de nuestra investigación consideramos que la inclusión del trabajo con software educativo en los procesos de enseñanza y de aprendizaje plantea la necesidad de tomar en cuenta modificaciones en relación con:

- El trabajo matemático de los alumnos, tanto en los problemas y tareas que se pueden abordar, como en las formas de abordarlos y en las posibles técnicas que se constituyen; al respecto diríamos que se incorporan actividades que no serían factibles para la enseñanza sin TIC. En líneas generales, este tipo de propuestas promueve un ambiente experimental en la clase de matemática que probablemente cambie la naturaleza de su aprendizaje.

- El trabajo de los profesores, considerando los nuevos conocimientos y nuevos espacios de decisión involucrados en la planificación y en la gestión de su proyecto de enseñanza. Mishra, P. y Koehler, M.J (2008) introducen el término Technological Pedagogical Content Knowledge (TPACK) como un modo de pensar acerca de los conocimientos que necesitan comprender los docentes para integrar efectivamente la tecnología en sus aulas. Ellos destacan que TPACK comprende no solo conocimiento de contenido ( $m a-$ temático en nuestro caso), pedagógico y tecnológico sino fundamentalmente comprender las complejas relaciones entre estas componentes del conocimiento. La necesidad de dirigir, sostenery apoyar desde la enseñanza los procesos de aprendizaje -individuales y colectivos-de los alumnos, lleva a Trouche (2004) 
a introducir la noción de orquestación instrumental. Esta noción contempla los espacios de decisión docente cuando se trabaja con la inclusión de las TICs, abarcando tanto aquellos vinculados a las tareas y las maneras de realizarlas como a los relacionados con los instrumentos y su organización para el trabajo individual y grupal.

Desde nuestra posición, y en el marco de la Didáctica de la Matemática, estas dos dimensiones de estudio, el trabajo matemático de los estudiantes y el trabajo de los profesores, adquieren cierta especificidad cuando se aborda un contenido particular. Para el caso de nuestra investigación la zona de estudio considerada fue el tema funciones cuadráticas $^{(2)}$. En particular, nos concentramos en el diseño de una primera situación pensada para dar lugar a la definición de dichas funciones vía la construcción de un modelo dinámico que sirva de soporte para estudiar la variación de magnitudes geométricas asociadas a la situación (utilizamos para ello el software educativo Geo Gebra).

Señalemos como antecedente importante a este proyecto que parte del equipo de investigación y algunos profesores que integran el espacio colaborativo habían desarrollado años atrás una propuesta de enseñanza para el estudio de la función cuadrática - sin uso de TICs- ${ }^{(3)}$. La situación que se analiza en este artículo retoma actividades planteadas en dicha propuesta identificando cuestiones que surgieron al anticipar el empleo de modelos dinámicos para el estudio de la covariación entre magnitudes geométricas (que se pueden interpretar en términos funcionales). Al estudiar esas covariaciones con tecnología, se produce necesariamente un proceso de discretización: ¿cuáles son los efectos de esa discretización y como pueden ser aprovechados para el aprendizaje?

Parte de nuestras reflexiones se centran en el pasaje del mundo geométrico-numérico continuo de las medidas geométricas al mundo geométrico-numérico de Geo Gebra, un mundo discreto con un funcionamiento interno (en parte desconocido) y con un funcionamiento observable por el usuario en la pantalla.

\section{Acerca de la conformación de un grupo colaborativo}

Como mencionamos antes, una característica del equipo de investigación es que está conformado por docentes/investigadores de la Universidad Pedagógica de Buenos Aires (UNIPE) y profesores de Escuela Secundaria en ejercicio. Compartimos la posición según la cual los profesores participan activamente en los procesos de transposición didáctica y, como precisa Brousseau «transponiendo saberes matemáticos deben apropiarse de las formas y las modificaciones actuales» (Brousseau, 1999:34). Los saberes especializados, tanto de los docentes como de los investigadores, aportan a la producción de un nuevo conocimiento didáctico, con necesarios procesos de negociación de preguntas y problemas de interés mutuo.

En ese sentido, la heterogeneidad del grupo posibilita una mirada integral al problema de la incorporación de las TICs al trabajo del aula. Creemos que, si bien algunos de los integrantes - por su experiencia y formación- privilegian los sucesos del aula mientras que otro grupo reconoce la necesidad de tender puentes con ciertos marcos teóricos, este proyecto de investigación - al estudiary seleccionar nuevos lenguajes, nuevas herramientas y nuevas técnicas del trabajo matemático- aporta al proceso de constitución del espacio colaborativo. Es en este marco de «igualdad de condiciones» -frente a la problemática de la incorporación de TICs a la enseñanza de las matemáticas- que nos planteamos como un objetivo compartido por todos los integrantes del grupo, la elaboración colectiva de materiales para docentes en los cuales se comunique, en el análisis a posteriori, la experiencia tanto de las actividades desarrolladas por los alumnos, como de la gestión del docente en las clases y de las interacciones que se generaron. Este análisis permitirá, eventualmente, el ajuste de los problemas/actividades/tareas acompañado con una descripción de posibles escenarios de clase. En general, las propuestas de trabajo mediado por TIC que están a disposición de los docentes de matemática, por ejemplo, aquellas que se encuentran 
en la web, no son acompañadas de una reflexión que contemple la complejidad didáctica que comporta su incorporación. La ausencia de este tipo de reflexiones puede dar lugar a un uso «ingenuo» $y$ acrítico de las TIC en el aula mediante propuestas que no dejan mucho margen para procesos de producción matemática de los alumnos.

\section{Datos contextuales de la situación en el país}

En nuestro país, el problema de incorporación de las TICs cobra relevancia significativa a partir de la provisión de netbooks en la escuela pública, una por alumno, una decisión política que ha sido tomada por varios gobiernos de países de Latinoamérica en los últimos años. En nuestro país el Programa Conectar Igualdad ${ }^{(4)}$ garantizó la distribución de las TICs en las escuelas, obligando a los distintos responsables de la enseñanza (profesores, especialistas, funcionarios) a pensar en qué sentido esta incorporación puede contribuir a una mejora de la educación secundaria.

Un cambio como el que hace posible la incorporación de las TICs necesita ser estudiado en sus diversas dimensiones. Como grupo sostenemos la necesidad de problematizar la inserción de las TICs a partir de propuestas de enseñanza contextualizadas y con temáticas específicas, que incluyan una explicitación de los cambios que aportan las TICs al repensar el qué y el cómo en forma conjunta. La fundamentación de las decisiones que se tomaron en el diseño de las propuestas y la explicitación del aporte de la tecnología, darían mejores condiciones para cambios profundos que incidan en los procesos de enseñanza.

\section{La zona de trabajo explorada: los modelos dinámicos para el estudio de una situación geométrica}

La presencia de programas de geometría dinámica -por ejemplo, Geo Gebra o Cabrí- pone en juego un universo de objetos y relaciones que son un modelo recortado de la geometría euclídea.

Como señala Laborde, en este tipo de programas

\section{[...] el trazado en la pantalla del dibujo de un objeto} geométrico tiene que conservar, en el transcurso del desplazamiento, las propiedades espaciales que dan cuenta de las propiedades geométricas de ese objeto, entonces tiene que hacerse mediante las primitivas geométricas (tales como punto medio, mediatriz, recta paralela, recta perpendicular, etc.). De esta manera, la exigencia de comunicar al programa un procedimiento geométrico de construcción permite caracterizar el objeto geométrico. (Laborde, 1998:38)

Con el uso de este tipo de software aparecen en el aula nuevos objetos: las figuras dinámicas. Estas figuras permiten configurar un nuevo tipo de tareas como, por ejemplo, el estudio de ciertas relaciones entre magnitudes geométricas vía modelos dinámicos que las representan.

Por otra parte, hay programas que agregan a la manipulación de figuras dinámicas la posibilidad de trabajar en el marco funcional. En estas interfaces es posible establecer distintos tipos de relaciones entre el plano geométrico, lo algebraico y lo funcional. En nuestra investigación seleccionamos un software con esta característica - Geo Gebra- para formular problemas que pongan en relación las variaciones visualizadas en el plano geométrico con la representación gráfica y la expresión algebraica de funciones que atrapan la covariación entre diferentes magnitudes de las figuras dinámicas.

Durante el proceso de diseño colectivo de actividades -en el cual priorizamos tanto la integración de las TICs como la producción matemática de los alumnos-se fue problematizando la relación entre 
un problema geométrico y el modelo dinámico que se construye para su estudio.

La construcción de un modelo dinámico para el estudio de una situación geométrica determinada necesita poner en juego relaciones geométricas e implica una serie de elecciones a través de las cuales se produce necesariamente un recorte en relación con la situación geométrica modelizada. Nos proponemos identificar algunas dimensiones de la complejidad didáctica que debe enfrentar la enseñanza al elegir, diseñar y orquestar este tipo de tareas.

En nuestro trabajo, un primer asunto a definir fue la elección de un tipo de problema matemático y el diseño de una propuesta de enseñanza que pusiera en juego un modelo dinámico y resultara fértil para un determinado grupo de alumnos.

En el seno del grupo colaborativo, la producción de actividades para el aula se realizó necesariamente en simultáneo con su análisis, con el despliegue de un espacio de posibles acciones de los alumnos y con el diseño de una orquestación para el desarrollo de las clases. Aportaron al diseño experiencias desarrolladas en forma independiente por algunos docentes integrantes del equipo ${ }^{(5)}$.

Durante este proceso de análisis, identificamos dos asuntos que desarrollaremos en este artículo:

- La relación entre el problema matemático y el modelo dinámico que se construye para estudiarlo. - La configuración del área de trabajo como variable didáctica.

\subsection{La relación entre el problema matemático y el modelo dinámico que se construye para estudiarlo}

En el proceso de modelización, el modelo construido recupera algunas relaciones matemáticas entre los elementos que caracterizan la situación modelizada.

Muy sucintamente diremos que un proceso de modelización supone en primer lugar recortar una cierta problemática frente a una realidad generalmente compleja en la que intervienen muchos más ele- mentos de los que uno va a considerar, identificar un conjunto de variables sobre dicha problemática, producir relaciones pertinentes entre las variables tomadas en cuenta y transformar esas relaciones utilizando a lgún sistema teórico-matemático, con el objetivo de producir conocimientos nuevos sobre la problemática que se estudia. (Sadovsky, 2005:27)

Durante este proceso se movilizan tanto conocimientos que permiten describir/caracterizar en términos matemáticos la situación a modelizar como las intenciones de quien modeliza, asociadas al futuro tratamiento matemático que se realizará en la búsqueda de una solución al problema que se está estudiando. En este sentido, diferentes modelos de la misma situación podrían poner en relieve distintos aspectos del problema, promover o limitar la elaboración de nuevas preguntas y obtener -0 no- respuestas a ciertos interrogantes.

Al producir modelos con un programa de geometría dinámica intervienen también decisiones en torno a los objetos y herramientas que ofrece el programa y a las opciones de configuración del área de trabajo. En muchas ocasiones estas decisiones están condicionadas por los conocimientos que cada usuario tiene del programa.

Las actividades diseñadas parten de una situación geométrica que involucra una familia infinita de rectángulos. Con cualquier modelo dinámico necesariamente se atrapan sólo finitos miembros de esa familia. Todo tratamiento digital implica una discretización de los objetos lo que lleva a la limitación del número de los mismos y de algunas de sus propiedades, aunque la interface con el usuario y la manipulación de los objetos en pantalla den la sensación de un continuo. Como señalan Morgan, C.; Mariotti, M. y Maffei, L (2009), las herramientas de arrastre aplicadas a una determinada figura producen una secuencia de imágenes y un cierto efecto dinámico que lleva a percibir un movimiento continuo, esto es, a percibir que la figura original se está desplazando de manera continua. En ese sentido, las figuras dinámicas constituyen un «modelo recortado» del problema geométrico en estudio. 
Queda a cargo de la enseñanza apuntalar la construcción de cierta racionalidad matemática para que los estudiantes distingan la situación geométrica de su modelo dinámico. ¿Cómo y en qué momento se trabaja con los estudiantes en torno a esta distinción? Hay una tensión para la enseñanza entre aprovechar la potencialidad de este tipo de modelos ${ }^{(6)}$-ya que permiten un trabajo matemático fértil de los estudiantes-y construir con ellos una mirada crítica acerca de sus limitaciones.

Valiéndonos de la noción de contrato didáctico (Brousseau, 1993) interpretamos que ante este contexto otras cuestiones se ponen en juego y es necesario repensar las reglas de juego en el aula, con el objetivo de hacer entrar a los estudiantes en las nuevas prácticas inherentes a las modelizaciones dinámicas y, por otro lado, lograr que ellos comprendan el recorte que implican estos modelos. Estas nuevas reglas del juego, mayormente implícitas, necesitan ser negociadas entre estudiantes y profesores para avanzar en un tipo de trabajo de mayor complejidad, mediado por TICs. Siguiendo los aportes del enfoque TPACK, no se concibe la tecnología como un «agregado», como algo adicional al conocimiento pedagógico-disciplinar. Con el replanteo del contrato didáctico se plasma como necesidad «reacomodar» nuestras prácticas. Estos tres conjuntos de saberes (matemáticos, pedagógicos y tecnológicos) se integran en las nuevas reglas del juego que construyen docentes y estudiantes. Esta problemática de la relación entre la situación geométrica y el modelo dinámico diseñado para estudiarla ha sido considerada en el diseño de un grupo de actividades. Con el propósito de precisar estas ideas presentamos a continuación tanto las actividades diseñadas como una fundamentación que da cuenta de las decisiones tomadas.

La situación a estudiar en el aula se relata oralmente a los estudiantes (antes de entregar un archivo del programa Geo Gebra para trabajar $\left.{ }^{(7)}\right)$ : «En un triángulo rectángulo isósceles cuyos lados iguales miden 11 , inscribimos rectángulos como lo muestra la figura». El relato se acompaña con dibujos estáticos en el pizarrón con dos o más rectángulos dibujados.

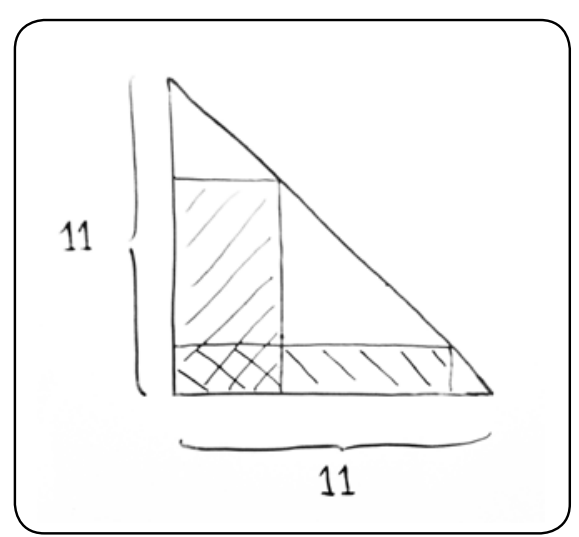

Figura 1.

Imagen del dibujo realizado en el pizarrón.

Las primeras preguntas del docente apuntan a algunas características de estos rectángulos: ¿Cuánto puede medir la base de cualquiera de estos rectángulos? ¿Cuántos rectángulos hay? Las respuestas a estas preguntas van precisando en el aula cuál es la familia infinita de rectángulos que será objeto de estudio. Esta manera de presentar la situación nos permite abordar la cuestión en juego como relativa a objetos matemáticos abstractos e infinitos.

Como parte de la orquestación instrumental se diseñó un archivo GeoGebra con características específicas (que serán explicadas en el apartado 4.2) para que los alumnos continúen trabajando con dicho archivo en el aula. Las posteriores interacciones de los estudiantes con el modelo Geo Gebra -necesariamente finito- que se va a construir permitirán al docente ir precisando cuál es el problema que se quiere estudiar.

Se les entrega a los alumnos un archivo $g g b$ y se les indica que en él hay un triángulo rectángulo isósceles cuyos lados iguales miden 11 , como el presentado en el pizarrón. Este momento marca el inicio de la actividad con el software. Se les pide a los estudiantes que construyan un rectángulo dinámico (inscripto en el triángulo). Esta construcción puede ser realizada de manera diferente por cada estudiante.

Una vez finalizada la construcción del rectángulo dinámico, el docente promoverá la búsqueda de 
rectángulos de ciertas dimensiones para que los estudiantes comiencen a establecer relaciones entre la situación geométrica y el modelo dinámico: ¿Todos pueden llegar al rectángulo que tenga como base 9,25? ¿y al de base 2,31?

Como veremos en el punto 4.2, el archivo fue preparado de modo que aparezcan sólo los rectángulos que tengan base con medidas que varían de 0 a 11 , con paso 0,05 . Algunas dimensiones requeridas pondrán de manifiesto la imposibilidad de alcanzar todas las medidas en el modelo dinámico y de este modo poner de manifiesto su finitud (las medidas alcanzadas son un subconjunto finito formado por los números decimales con solo dos cifras, terminando en 00 5).

Para abrir el juego a la relación entre las magnitudes de área y la longitud de un lado el docente preguntará por algunos casos particulares: ¿Cuál será el área del rectángulo cuando la base mide 2? ¿Ycuando la base mide 8, 45? ¿Ycuando la base mide 2,31 ?. Se espera que los estudiantes se apoyarán en las construcciones ya realizadas y el docente mostraría la herramienta «Polígono» que ofrece el software para el cálculo de áreas.

En algunos casos el rectángulo requerido puede obtenerse por arrastre y en la pantalla se visualiza el valor de su área. En cambio, la pregunta sobre el área del rectángulo de base de medida 2,31 requerirá que los estudiantes recurran a otras relaciones para responderla. El modelo dinámico - tal cual está configurada el área de trabajo-no permite obtener una base de esa longitud; sería necesario «salirse» del modelo dinámico y encontrar otras formas de acceder a la respuesta, sin apoyarse en los resultados que automáticamente ofrece el programa.

Al interrogar acerca de valores que están fuera del rango que reconoce la configuración del área de trabajo del archivo, se propicia que los estudiantes pongan en juego relaciones y propiedades que les permitan calcular el área, en particular identificar cuál es la relación entre los dos lados de cada rectángulo de la familia.

Desde una perspectiva más abarcativa del proceso de incorporación de las TICs al trabajo matemático de los estudiantes, un recorrido como éste habilita a empezar a discutir en el aula la diferencia entre la situación geométrica a estudiar y el modelo dinámico que se construye para estudiarla. En particular las preguntas analizadas hasta aquí sobre los valores presentes y o ausentes en el modelo son una oportunidad para aproximarse a la idea matemática de modelización. Las discusiones que se pueden propiciar permitirían comprender más profundamente las limitaciones de la tecnología digital para manipular con números en «un continuo», o con números irracionales. Gestar una tal discusión así pone de relieve la trama entre conocimientos matemáticos y funcionamiento del programa con la que debe maniobrar el docente (TPACK), más allá de la tensión que debe asumir entre destinar tiempo a plantear problemáticas poco exploradas pero interesantes y focalizar en el problema de estudio.

\subsection{La configuración del área de trabajo como variable didáctica}

Elegimos la noción de variable didáctica para considerar las posibles configuraciones del área de trabajo que el Geo Gebra habilita y las consecuencias que ellas pueden tener en las producciones de los estudiantes. En el sentido que la Teoría de Situaciones considera, las variables didácticas «son elementos de la situación que se pueden modificar desde la enseñanza para provocar un cambio en las formas de resolución de los estudiantes» (Brousseau, 2007:32).

En todo entorno de geometría dinámica ciertas características del área de trabajo están predeterminadas por valores que fueron asignados, en principio, por los desarrolladores del entorno; algunas de esas variables pueden ser modificadas por el usuario. En el entorno Geo Gebra aparecen identificadas como «0pciones» en la barra de tareas. Se puede modificar, por ejemplo, la cantidad de cifras que se consideran para el redondeo, el tamaño de la cuadrícula en la(s) vista(s) gráfica(s) y el uso o no de imanes. 
Ahora bien, en función del problema elegido, para diseñar una situación de enseñanza - lo cual incluye pensar en su orquestación efectiva en el aula- hemos creído conveniente considerar si se fijan o no ciertas opciones de configuración. Estas opciones afectarán tanto el trabajo individual de cada alumno como el trabajo colectivo en el aula. En nuestro proceso de construcción de una situación de enseñanza introductoria de las funciones cuadráticas hemos podido profundizar en torno a tres interrogantes:

- ¿Qué hay detrás de lo que se ve en la pantalla de Geo Gebra?

- ¿Cómo «atrapar» un objeto específico en la pantalla? - ¿Qué trae aparejado el uso de diferentes configuraciones del área de trabajo en el aula?

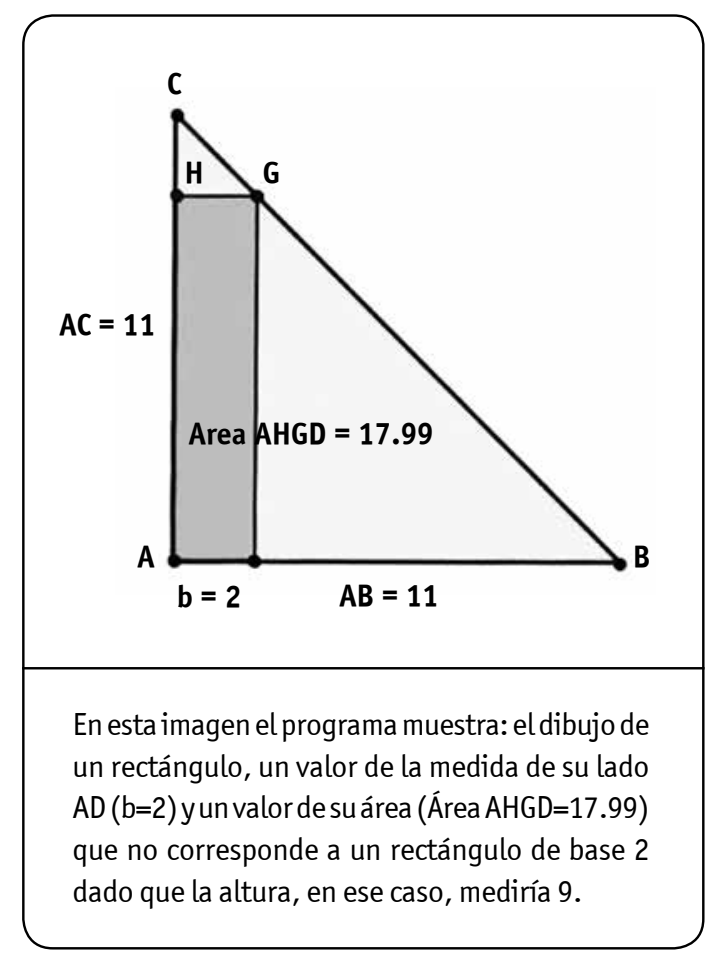

Figura 2.

Captura de pantalla de Geo Gebra del área de trabajo de un alumno.

\subsection{1. ¿Qué hay detrás de lo que se ve en la pantalla de Geo Gebra?}

Como ya dijimos, la situación de enseñanza presentada en el apartado 4.1 fue objeto de algunas experiencias preliminares en las cuales los estudiantes trabajaban con la configuración habitual del programa, sin ningún archivo preparado por nosotros. Los hechos referidos por los docentes, serán presentados aquí como medio para precisar nuestra pregunta. Y fueron al mismo tiempo detonantes de las decisiones que finalmente tomamos en el diseño. Los alumnos estaban estudiando el área de rectángulos de perímetro fijo. En particular, consideraron los rectángulos inscriptos en un triángulo rectángulo isósceles cuyos catetos miden $11 \mathrm{~cm}$. Se les pidió calcular el área de un rectángulo de base 2. Usando Geo Gebra, un estudiante llega a la siguiente pantalla:

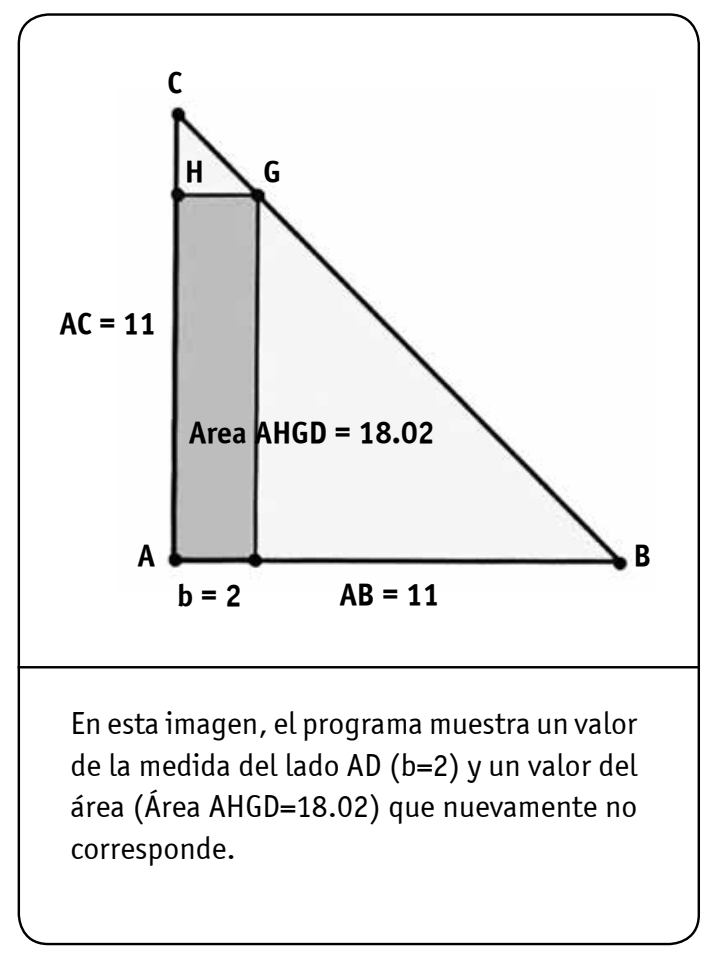

Figura 3.

Captura de pantalla de Geo Gebra del área de trabajo de un alumno. 
En ambos ejemplos, el resultado mostrado por el programa como valor del área del rectángulo no se corresponde con el valor que se muestra para la medida de $A D$, dado que para un rectángulo con un lado midiendo 2 inscripto en un triángulo isósceles cuyos catetos miden 11, el área es 18.

Este fenómeno ocurre debido a que el programa Geo Gebra hace aproximaciones para mostrar la información -según la cantidad de cifras determinadas por el usuario- pero utiliza mayor precisión en los cálculos. En el aula donde ocurrieron estos hechos estaban trabajando con una aproximación a dos decimales en la pantalla, y en los dos casos los estudiantes habían llegado a un rectángulo con el lado $A D$ con medida diferente de 2, pero con una diferencia no detectable en las dos primeras cifras decimales. Sin embargo, estas diferencias influyeron en el cálculo del área que hizo el programa, devolviendo valores que difieren en las dos primeras cifras decimales. Simulando esta situación en nuestra investigación llegamos a una pantalla con cuatro decimales (ver Figura 4).

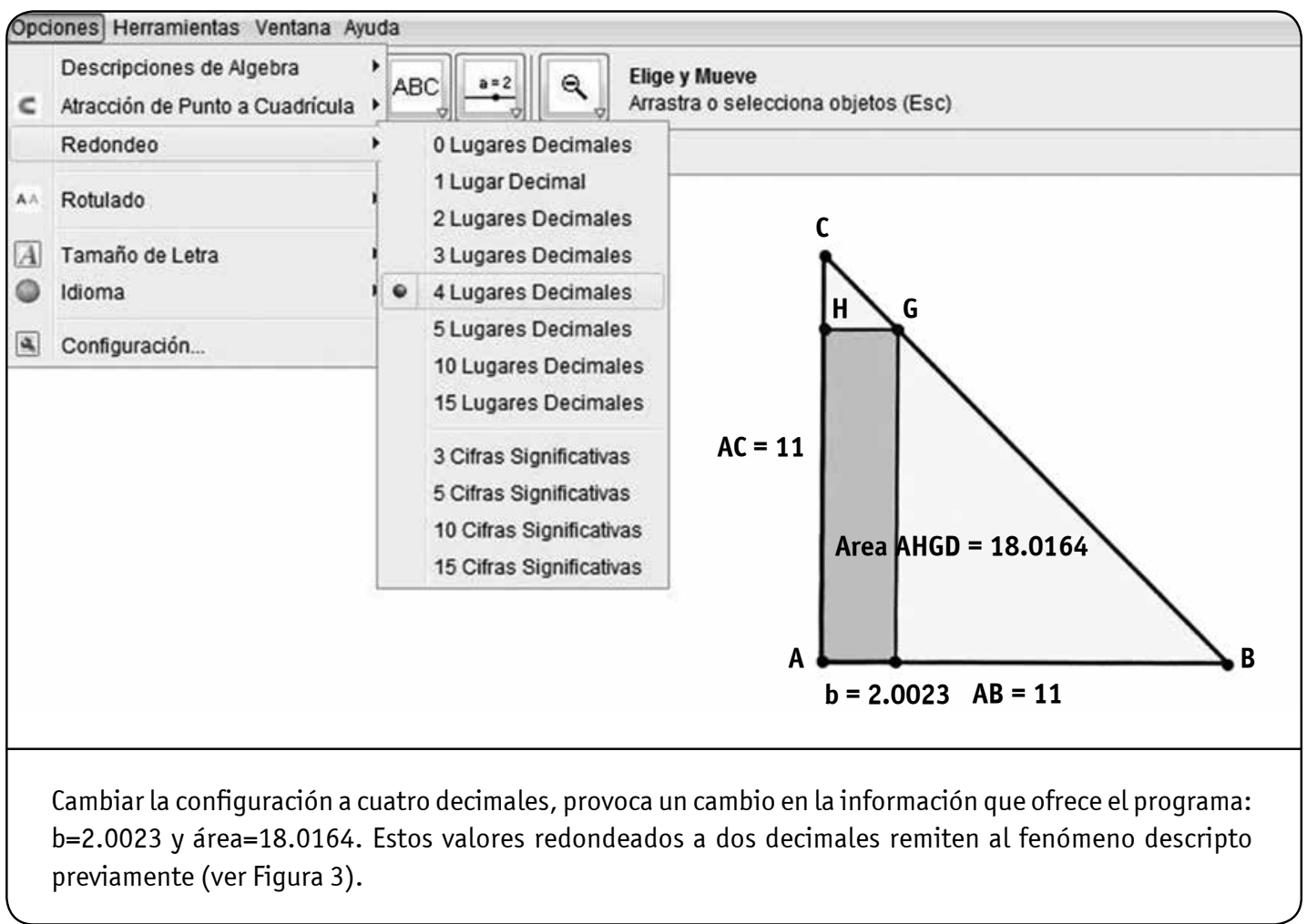

Figura 4.

Captura de pantalla de Geo Gebra que muestra la elección de cifras decimales para redondeo.

Como vemos en este ejemplo, aumentar los decimales que el programa muestra, no resuelve el problema del ajusteya mencionado en los ejemplos anteriores. Estos ejemplos nos alertan sobre las implicancias que pueden tener las aproximaciones de cálculo que realiza el programa durante el proceso de resolución de un problema. En este sentido, si no se toman algunas precauciones respecto a la configuración del archivo con el cual trabajarán los estudiantes, posiblemente aparezcan «alteraciones», como 
las señaladas antes, que podrían obstaculizar el desarrollo de la tarea.

Pensando en una entrada al estudio de la variación de magnitudes vía modelos dinámicos, estas «alteraciones» pueden provocar que los alumnos prescindan del modelo para responder las preguntas que se les planteen, tal como ocurrió en la clase que recién mencionamos: los estudiantes comenzaron a desconfiar del programa y decidieron continuar con el problema prescindiendo del uso de GeoGebra En pos de «minimizar las alteraciones» creímos necesario diseñar actividades interviniendo la configuración a utilizar en la clase durante las primeras aproximaciones de los alumnos con estas formas de trabajo: consideramos pertinente que, para las variables que el programa tendrá en cuenta para realizar cálculos, el valor que se muestre en la pantalla sea el que efectivamente toma el software como dato de entrada para la realización de dichos cálculos. Estas decisiones, junto a las que eventualmente surjan en la situación de clase, son parte del proceso de orquestación instrumental.

Esta experiencia nos invita a pensar prospectivamente de qué manera ir haciendo explícito en el aula estas particularidades del trabajo con el soft, y al mismo tiempo pensar situaciones de enseñanza que aprovechen estas aparentes disfuncionalidades a favor del enriquecimiento del trabajo matemático de los alumnos.

\subsection{2. ¿Cómo «atrapar»} un objeto específico en la pantalla?

Quienes hayan trabajado con figuras dinámicas, habrán experimentado la dificultad de «atrapar», mediante el arrastre, un objeto particular. Retomamos las producciones de los estudiantes, mencionadas anteriormente y a partir de ellas precisamos esta idea.

Tratando de «atrapar» un rectángulo con medida $A D=2$, los estudiantes a través del movimiento de arrastre ubicaban el extremo $D$ del segmento en distintas posiciones, de tal manera que el software mostraba en pantalla el valor $b=2$. Sin embargo, la medida que efectivamente asignaba el software para ese segmento era otra.

Esta situación nos abre otro tipo de problemática referida también a las características de la interface de comunicación que ofrece el programa. Se trata ahora de considerar las relaciones entre:

- los movimientos físicos que se realizan con la mano para ubicar la posición del puntero del mouse, y - los diversos valores que asigna el software a las magnitudes que dependen de la posición del puntero.

En nuestro diseño, optamos finalmente por una configuración tal que los valores delárea del rectángulo que aparecían en la pantalla correspondieran al valor de la base que se mostraba, para todo rectángulo al que se llegaba por arrastre. Para lograrlo en GeoGebra, decidimos regular el movimiento del mouse mediante la determinación de una cuadrícula suficientemente pequeña y el uso de atractores de puntos a cuadrícula. Para ello configuramos el área de trabajo en un archivo Geo Gebra que entregaríamos a cada estudiante para que trabaje.

\subsection{3. ¿Qué trae aparejado el uso de diferentes configuraciones del área de trabajo en el aula?}

Las dos problemáticas explicadas en los apartados anteriores ponen en evidencia la diversidad de configuraciones del área de trabajo que podrían convivir simultáneamente en una clase.

Esta heterogeneidad no parece la más indicada para las primeras experiencias con estas formas de trabajo, tanto para los alumnos como para los docentes. Particularmente, adoptar criterios comunes a toda la clase para configurar elárea de trabajo puede ser una buena estrategia para minimizar las consecuencias de las aproximaciones numéricas que inevitablemente devuelve el programa en sus cálculos.

No obstante, queremos destacar que un escenario interesante al cual apuntar desde la enseñanza es la construcción de un tipo de racionalidad en el aula que permita a los estudiantes poder interactuar: 
- con el software, interpretando y evaluando críticamente las devoluciones que éste ofrece; particularmente analizando si una respuesta es plausible $o$ incoherente para el problema en cuestión.

- con la diversidad de configuraciones del área de trabajo que podrían circular en la clase; en este caso dialogando con las distintas respuestas y participando en un trabajo colectivo de comparación y contrastación necesariamente sostenido y coordinado porel docente.

El conocimiento de la lógica del funcionamiento del software iría brindando autonomía para que los mismos alumnos sean quienes decidan cuál es la configuración más apropiada a cada situación planteada; incluso, podrían decidir en forma consciente adoptar la configuración por defecto que ofrece el software e interpretar los resultados que devuelve en el marco de las aproximaciones que realiza el programa.

Desde nuestro punto de vista el tránsito hacia un mayor nivel de autonomía en el trabajo de los estudiantes, no se da espontáneamente; por el contrario, para que se concrete debería formar parte del proyecto de enseñanza en términos de acciones que permitan hacer evolucionar «una entrada controlada y homogénea» hacia formas de trabajo más abiertas y autónomas.

\section{Reflexiones finales}

Las problemáticas presentadas en este artículo dan cuenta de la complejidad subyacente a la intención de hacer entrar a los alumnos en un tipo de trabajo con problemas geométricos estudiados vía modelos dinámicos, en los cuales la medida de ciertas magnitudes cobra un valor importante. En este artículo abordamos esa complejidad desde la perspectiva del diseño de actividades y centrándonos en dos aspectos.
Por un lado, la responsabilidad de la enseñanza de trabajar en el aula explícitamente las relaciones y diferencias entre una situación geométrica y un modelo dinámico que se construye para estudiarla. En particular la idea de que los modelos dinámicos atrapan siempre sólo finitos elementos de una familia infinita de figuras.

Por otro lado, nos detuvimos a estudiar cómo decisiones relativas a la configuración del área de trabajo condicionan la producción matemática que los estudiantes pueden llevar adelante con el programa.

En otro orden digamos que las cuestiones revisadas en este artículo son propias del funcionamiento de todo software de geometría dinámica. Hemos tomado ejemplos referidos al programa Geo Gebra que sabemos se replican en otros con especificidades que van mutando a partir de las actualizaciones de los mismos.

Desde nuestra posición, la producción de propuestas que contemplen características particulares del software no es independiente de la actividad matemática que se busca instalar en el aula. Es un desafío para la didáctica concebir escenarios en los cuales ciertas particularidades del software se aprovechen para convocar a los estudiantes a un trabajo matemático más sustantivo. Como lo señala el modelo TPACK, una integración genuina de las TIC requiere de la enseñanza poner en juego de manera relacionada tres tipos de conocimiento: disciplinar, pedagógico y tecnológico.

Nuestro trabajo de investigación no busca ofrecer soluciones generales ni modelos de enseñanza. Integrar las TICs en las prácticas de enseñanza de Matemática en modo cotidiano implica enfrentar problemas didácticos más complejos, no más sencillos. Es necesario repensar las propuestas en su complejidad y esta es una tarea en la cual los equipos de investigación podemos colaborar. 


\section{Notas}

(1) La investigación se inscribe en los proyectos: «La transformación del trabajo matemático en el aula del secundario a partir de la integración de las TICs: un problema didáctico pensado con docentes» en el ámbito de la UNIPE (Universidad Pedagógica de la Provincia de Buenos Aires, Argentina) durante el año 2012 y «La integración de la TIC en la enseñanza de funciones en la escuela secundaria. Diseño de actividades y análisis del trabajo en el aula a cargo de un equipo integrado por docentes e investigadores», proyecto PICT0-2012-0040 (PICT0: Proyectos de Investigación Científica y Tecnológica Orientados dependiente de La Agencia Nacional de Promoción Científica y Tecnológica, Ministerio de Ciencia, Tecnología e Innovación Productiva de la República Argentina.

(2) El estudio de funciones cuadráticas, en nuestro país, es abordado en $3^{\circ} 04^{\circ}$ año de la escuela secundaria, que son el décimo o undécimo año de escolaridad obligatoria (en general, se trata de estudiantes que tienen entre 15 y 17 años de edad).

(3) Documento curricular del ministerio de educación del Gobierno de la Ciudad de Buenos Aires: Matemática, función cuadrática parábola y ecuación de segundo grado http://estatico.buenosaires. gov.ar/areas/educacion/curricula/media/matematica/matematica-cuadratica.pdf

(4) Hasta el año 2015, el estado argentino -en el marco de dicho programa- entregó más de dos millones de netbooks a estudiantes y docentes de escuelas públicas secundarias, de educación especial y de Institutos de Formación Docente.

(5) En el año 2013 tres profesoras del equipo de investigación llevaron a sus aulas algunas de esas propuestas enriquecidas con el aporte de las TIC y algunos ejemplos que presentamos en este artículo provienen de dichas experiencias.

(6) Entre otras potencialidades de esos modelos dinámicos podemos señalar la «visualización» de una figura en movimiento y la inmediatez de las repuestas que da el soft en el cálculo de las medidas de las magnitudes en juego, para cada estado de la figura dinámica.

(7) En adelante los archivos del programa Geo Gebra serán nombrados: «archivos ggb».

\section{Referencias bibliográficas}

Artigue, M. (2002). Learning mathematics in a CAS environment: the genesis of a reflection about instrumentation and the dialectics between technical and conceptual work. International Journal of Computers for Mathematical Learning, 7 (3), 245-274.

Artigue, M. (2013). L'impact curriculaire des technologies sur l'éducation mathématique. Cuadernos de Investigación y Formación en Educación Matemática, 8(11), 295-305. Costa Rica: Universidad de Costa Rica.

Balacheff, N. (2000). Entornos informáticos para la enseñanza de las matemáticas: complejidad didáctica y expectativas. En N. Gorgorió, A. Deulofeu y A. Bishop (coordinadores), Matemáticas y educación. Retos y cambios desde una perspectiva internacional. Barcelona: Editorial GRAO.

Brousseau, G. (1993). Fundamentos y métodos de la Didáctica de la Matemática, Universidad Nacional de Córdoba, Traducción FAMAF, Serie B, Trabajos de Matemática, 19.

Brousseau, G. (1999). Educación y didáctica de las matemáticas. Educación Matemática, 12 (1), 5-38.

Brousseau, G. (2007). Iniciación al estudio de la teoría de las situaciones didácticas. Libros del Zorzal. Buenos Aires.

Hoyles, C. y Lagrange, J.B. (2010) (Eds.) Mathematics Education and Technology-Rethinking the Terrain. The 17th ICMI Study. New York: Springer

Hoyles, C.; Noss, R.; Kent, P. (2004). On the integration of digital technologies into mathematics classrooms. International Journal of Computers for Mathematical Learning, 9 (3), 309-326. 
Laborde, C. (1998). Cabri-Géomètre o una nueva relación con la geometría. En L. Puig \& J. Calderón (Eds.), Investigary Enseñar. Variedades de la Educación Matemática, 33-48. Bogotá: Una Empresa Docente. Lagrange, J.B. (2000). L'intégration d'instruments informatiques dans l'enseignement: une approche par les techniques. Educational Studies in Mathematics, 43 (1), 1-30.

Morgan, C.; Mariotti, M. y Maffei, L. (2009). Representation in Computational Environments: Epistemological and Social Distance. International Journal of Computers for Mathematical Learning, 14 (3), 241-263.

Mishra, P. y Koehler, M.J. (2008). Introducing Technological Pedagogical Content Knowledge. Trabajo presentado en el Annual Meeting of the American Educational Research Association, New York City. Recuperado el 11 de Agosto de 2014, de: http://punya.educ.msu.edu/presentations/AERA2008/ MishraKoehler_AERA2008.pdf

Rockwell, E. (1995). La escuela cotidiana. Fondo de Cultura Económica. México.

Sadovsky, P. (2005). Enseñar matemáticas hoy. Miradas, sentidos y desafíos. Libros del Zorzal. Buenos Aires.

Trouche, L. (2004). Managing the complexity of human/machine interactions in computerized learning environments: guiding students' command process through instrumental orchestrations. International Journal of Computers for Mathematical Learning, 9 (3), 281-307. 